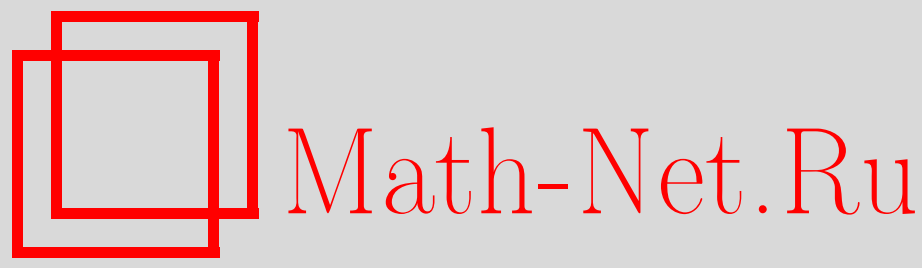

Г. А. Мартынов, Степенная и экспоненциальная асимптотика корреляционных функций, ТМФ, 2008, том 156 , номер 3, 454-464

DOI: https://doi.org/10.4213/tmf6259

Использование Общероссийского математического портала Math-Net.Ru подразумевает, что вы прочитали и согласны с пользовательским соглашением http://www.mathnet.ru/rus/agreement

Параметры загрузки:

IP : 18.209 .158 .208

26 апреля 2023 г., 07:11:21

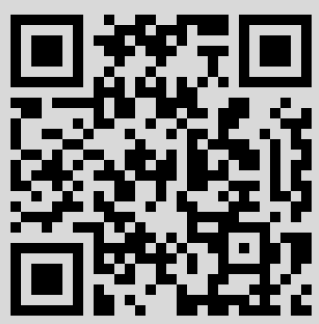




\title{
СТЕПЕННАЯ И ЭКСПОНЕНЦИАЛЬНАЯ АСИМПТОТИКА КОРРЕЛЯЦИОННЫХ ФУНКЦИЙ
}

\begin{abstract}
С помощью уравнения Орнштейна-Цернике получены два асимптотических уравнения, одно из которых описывает экспоненциальную, другое - степенную асимптотику общей корреляционной функции $h(r)$. Показано, что экспоненциальная асимптотика справедлива только на ограниченном отрезке расстояний $l<r<L$. При $r>L$ всегда реализуется степенная асимптотика, воспроизводящая вид потенциала взаимодействия. При этом в разреженных газах с уменьшением плотности $L \rightarrow l$, экспоненциальная асимптотика исчезает и остается только степенная асимптотика. Наоборот, с приближением к критической точке $L \rightarrow \infty$ и область применимости экспоненциальной асимптотики возрастает до бесконечности.
\end{abstract}

Ключевые слова: асимптотика, корреляционные функции, уравнение ОрнштейнаЦернике.

\section{1. ВВЕДЕНИЕ}

Как известно, все макроскопические параметры системы определяются состоянием корреляционной сферы, представляющей собой флуктуацию плотности на фоне пространственно однородной жидкости. В статистической механике распределение плотности $\rho(r)$ внутри этой сферы описывается общей корреляционной функцией $h(r)=G(r)-1$, где $G(r)$ - двухчастичная функция распределения. Радиус сферы $R$ (т.е. размер флуктуации) зависит от того, как быстро убывает функция $h(r)$ при $r \rightarrow \infty$, т.е. от ее асимптотики.

В настоящее время известны две асимптотики общей корреляционной функции. Одна из них - экспоненциальная, означающая, что в пределе больших расстояний функция $h(r) \rightarrow A e^{-\lambda r} / r$. Другая - степенная, согласно которой функция $h(r) \rightarrow \varphi(r) \sim 1 / r^{k}$ при $r \rightarrow \infty$, где $\varphi(r)=\Phi(r) / \theta$ - безразмерный потенциал взаимодействия частиц, $\theta=k_{\mathrm{B}} T$ - температура. В дальнейшем для определенности будем считать $k=6$, что характерно для большинства веществ.

Формула для степенной асимптотики была получена в [1] путем суммирования наиболее дальнодействующих диаграмм, т.е. методом, который, строго говоря, справедлив только в области сходимости вириальных рядов. Как было показано в [2], эта

* Институт физической химии РАН, Москва, Россия. E-mail: g2302@migmail.ru 
область охватывает лишь часть фазовой диаграммы жидкости. Поэтому возникает вопрос: совпадает ли область применимости степенной асимптотики с областью сходимости вириальных рядов?

Формула, определяющая экспоненциальную асимптотику, лежит в основе так называемой классической теории критических явлений [3]. Она была получена Орнштейном и Цернике еще в 1914 г. в результате приближенного решения предложенного ими же уравнения. Однако в дальнейшем более тщательный анализ показал, что это решение, строго говоря, справедливо только в случае "оборванных" потенциалов, для которых $\Phi(r) \equiv 0$ при $r>l$ [4], [5]. При этом, как это следует из результатов численных расчетов, значения констант $A$ и $\lambda$ в формуле для экспоненциальной асимптотики сильно зависят от того расстояния $l$, на котором производится обрыв интегралов, определяющих значения этих констант. Если же область интегрирования расширить до бесконечности, интегралы, определяющие константы $A$ и $\lambda$, будут расходиться (см. ниже раздел 3). Поэтому возникает вопрос: какой смысл имеет экспоненциальная асимптотика для реальных систем, для которых условие $\Phi(r) \equiv 0$ при $r>l$ никогда не выполняется?

Наконец, любая функция, казалось бы, может иметь только одну асимптотику. Как согласовать это очевидное утверждение с тем, что по современным представлениям $h(r)$ имеет две асимптотики?

Настоящая статья посвящена рассмотрению этих вопросов. При этом мы будем исходить из уравнения Орнштейна-Цернике (ОЦ), которое, как известно, является эквивалентом распределения Гиббса [6], [7]. С его помощью мы найдем вид асимптотического уравнения ОЦ, описывающего как степенную, так и экспоненциальную асимптотику, а затем обсудим следствия, вытекающие из полученных соотношений.

\section{2. СТЕПЕННАЯ АСИМПТОТИКА}

2.1. Уравнение Орнштейна-Цернике. Как известно, уравнение ОЦ

$$
h\left(r_{13}\right)=C\left(r_{13}\right)+\rho \int_{V} C\left(r_{13}\right) h\left(r_{32}\right) d \vec{r}_{3},
$$

где $\rho$ - плотность числа частиц, устанавливает связь между двумя неизвестными функциями: общей корреляционной функцией $h(r)$ и прямой корреляционной функцией $C(r)$. Чтобы преобразовать его в замкнутое уравнение, необходимо задать конкретный вид $C(r)$. Обычно эту функцию подбирают из условия наилучшего соответствия функции $h(r)$ результатам численного эксперимента. При этом, естественно, возникает определенный произвол в выборе аппроксимирующей функции, что делает выводы теории недостаточно надежными. Однако по определению [8] этот произвол ограничен тем, что

$$
C(r)=h(r)-w(r)+B[h]
$$

где $h(r) \equiv G(r)-1=e^{-\varphi(r)+w(r)}-1, w(r)$ - термический потенциал, $B[h]-$ бриджфункционал. В формуле (2) функции $h(r)$ и $w(r)$ ни при каких аппроксимациях меняться не могут, так как они являются прямым следствием распределения Гиббса. 
В то же время бридж-функционал $B[h]$ задается бесконечным диаграммным рядом, коэффициенты которого нам неизвестны [8]. Поэтому мы вынуждены аппроксимировать его некоторой функцией $B(h)$. Но при этом из диаграммного ряда следует, что $B[h] \sim w^{2}(r)$ на больших расстояниях $r$, где согласно условиям ослабления корреляций $w(r) \rightarrow 0$ при $r \rightarrow \infty$ [8]. Поэтому бридж-функционал вносит в асимптотику корреляционных функций вклад второго порядка малости, и, следовательно, в данном случае им можно пренебречь. В результате формулы, описывающие асимптотику корреляционных функций, оказываются однозначным следствием уравнения ОЦ (и тем самым однозначным следствием распределения Гиббса).

Произведя в (1) интегрирование по углам, получим

$$
\tilde{h}(r)=\widetilde{G}(r)+2 \pi \rho \int_{0}^{\infty} \widetilde{G}(x) d x \int_{|r-x|}^{r+x} \tilde{h}(y) d y
$$

где $\tilde{h}(r)=r h(r), \widetilde{C}(r)=r C(r)$. Чтобы избавиться в правой части полученного выражения от модуля $|r-x|$, продифференцируем его по $r$. В результате исходное уравнение ОЦ (1) преобразуется в следующее уравнение [8]:

$$
\frac{1}{2 \pi \rho} \frac{d}{d r}(\tilde{h}(r)-\widetilde{C}(r))=-\int_{0}^{r} \widetilde{C}(x) \tilde{h}(r-x) d x+\int_{0}^{\infty}[\widetilde{C}(x) \tilde{h}(r+x)+\widetilde{C}(r+x) \tilde{h}(x)] d x .
$$

Такое представление удобно тем, что оно содержит только одинарные интегралы. Как и (1), оно определяет общую корреляционную функцию $h(r)$ при $0<r<\infty$. Наша задача - найти с помощью (3) уравнение, описывающее ту же функцию $h(r)$, но только в пределе больших расстояний.

2.2. Асимптотическое уравнение ОЦ. Заметим, что согласно (2) выражение, стоящее в (3) под знаком производной, стремится к $h-C=w-B \rightarrow w$ при $r \rightarrow \infty$, так как $B \sim w^{2}$. В результате в этом пределе производная в левой части (3) становится равной $d(\widetilde{w}) / d r$, где $\widetilde{w}=r w(r)$.

Рассмотрим теперь первый интеграл в правой части (3). Пусть $r=\xi$ - расстояние, начиная с которого функции $C(r)$ и $h(r)$ одновременно становятся практически равными нулю. Очевидно, что в этом случае интеграл

$$
\int_{\xi}^{r-\xi} \widetilde{C}(x) \tilde{h}(r-x) d x
$$

также будет равен нулю. Поэтому в (3) первый интеграл можно представить в виде

$$
\begin{aligned}
\int_{0}^{r}(\ldots) d x & =\left(\int_{0}^{\xi}+\int_{\xi}^{r-\xi}+\int_{r-\xi}^{r}\right)(\ldots) d x \rightarrow \\
& \rightarrow \int_{0}^{\infty} \widetilde{C}(x) \tilde{h}(r-x) d x+\int_{0}^{\infty} \widetilde{C}(r-x) \tilde{h}(x) d x
\end{aligned}
$$

где в интеграле $\int_{r-\xi}^{r}(\ldots) d x$ мы сделали замену $x^{\prime}=r-x$, после чего устремили $\xi \rightarrow \infty$. 
Преобразуя второй интеграл в (3) и учитывая, что согласно (2)

$$
\widetilde{C}(r) \rightarrow-\tilde{\varphi}(r), \quad \tilde{h}(r) \rightarrow-\tilde{\varphi}(r)+\widetilde{w}(r),
$$

где $\tilde{\varphi}=r \varphi(r)$, при $r \rightarrow \infty$ получаем

$$
\int_{0}^{\infty}(\ldots) d x \rightarrow \int_{0}^{\infty}\{\widetilde{C}(x)[-\tilde{\varphi}(r+x)+\widetilde{w}(r+x)]+\tilde{h}(x)[-\tilde{\varphi}(r+x)]\} d x .
$$

В результате уравнение ОЦ (3) преобразуется в асимптотическое уравнение ОЦ [8]

$$
\begin{aligned}
& \frac{d \widetilde{w}(r)}{d r}-2 \pi \rho \int_{0}^{\infty} \widetilde{C}(x)[\widetilde{w}(r+x)-\widetilde{w}(r-x)] d x= \\
& \quad=-2 \pi \rho \int_{0}^{\infty}[\widetilde{C}(x)+\tilde{h}(x)](\tilde{\varphi}(r+x)-\tilde{\varphi}(r-x)) d x,
\end{aligned}
$$

определяющее термодинамический потенциал $\widetilde{w}(r)=r w(r)$ по заданному потенциалу взаимодействия

$$
\tilde{\varphi}(r)=r \varphi(r) \rightarrow-r \cdot 4 \frac{\varepsilon}{\theta}\left(\frac{\sigma}{r}\right)^{6}
$$

и заданным значениям функций $h(r)$ и $C(r)$ (здесь мы для наглядности ввели обозначения, справедливые только в случае потенциала Леннарда-Джонса).

Уравнение (7) содержит две неизвестные функции $h(r)$ и $C(r)$, значения которых определяются исходным уравнением ОЦ. Подставляя их в (7), мы преобразуем это уравнение в линейное интегро-дифференциальное уравнение с правой частью для определения асимптотических значений термического потенциала $w(r)$ по заданным значениям $h(r)$ и $C(r)$. Его общее решение состоит из двух слагаемых: частного решения этого уравнения с правой частью и решения соответствующего однородного уравнения [8]

$$
\frac{d \widetilde{w}(r)}{d r}-2 \pi \rho \int_{0}^{\infty} \widetilde{C}(x)[\widetilde{w}(r+x)-\widetilde{w}(r-x)] d x=0 .
$$

Рассмотрим сначала частное решение.

2.3. Степенная асимптотика. Подставляя соотношение (8) в (7), получаем

$$
\begin{aligned}
& \frac{d \widetilde{w}(r)}{d r}-2 \pi \rho \int_{0}^{\infty} \widetilde{C}(x)[\widetilde{w}(r+x)-\widetilde{w}(r-x)] d x= \\
& \quad=2 \pi \rho \cdot 4 \frac{\varepsilon}{\theta} \sigma^{6} \int_{0}^{\infty}[\widetilde{C}(x)+\tilde{h}(x)]\left(\frac{1}{(r+x)^{5}}-\frac{1}{(r-x)^{5}}\right) d x .
\end{aligned}
$$

Разлагая правую часть (10) в ряд по степеням $1 / r$ и ограничиваясь первым ненулевым членом ряда, представим (9) в виде

$$
\frac{d \widetilde{w}(r)}{d r}-2 \pi \rho \int_{0}^{\infty} \widetilde{C}(x)[\widetilde{w}(r+x)-\widetilde{w}(r-x)] d x=2 \pi \rho\left[C_{2}+h_{2}\right] \cdot 10 \cdot 4 \frac{\varepsilon}{\theta}\left(\frac{\sigma}{r}\right)^{6},
$$


где

$$
C_{2}=\int_{0}^{\infty} C(r) r^{2} d r, \quad h_{2}=\int_{0}^{\infty} h(r) r^{2} d r .
$$

Решение полученного уравнения будем искать в виде $w=\chi / r^{6}$. Подставляя это выражение в левую часть уравнения и разлагая подынтегральное выражение в ряд по степеням $1 / r$, находим

$$
\chi=\frac{C_{2}+h_{2}}{1-4 \pi \rho C_{2}} \cdot 4 \frac{\varepsilon}{\theta} \sigma^{6}, \quad w=-\frac{C_{2}+h_{2}}{1-4 \pi \rho C_{2}} \varphi(r) .
$$

Как известно, изотермическая сжимаемость [8]

$$
\kappa_{\theta}=1+4 \pi \rho h_{2}=\frac{1}{1-4 \pi \rho C_{2}} .
$$

С учетом этих соотношений находим

$$
w(r)=-\left[\kappa_{\theta}^{2}-1\right] \varphi(r), \quad h(r)=-\kappa_{\theta}^{2} \varphi(r) .
$$

Это хорошо известные формулы [1]. Они показывают, что в системах нейтральных частиц, как и в случае кулоновских систем, на больших расстояниях происходит экранировка потенциала $\varphi(r)$, создаваемого частицей, расположенной в центре корреляционной сферы. Однако при $\rho \rightarrow 0$ сжимаемость $\kappa_{\theta} \rightarrow 1$, в результате чего в разреженных газах экранировка исчезает. В этом случае вклад коллективной компоненты, описываемый термическим потенциалом $w(r)$, становится равным нулю, а общая корреляционная функция $h(r)$ ведет себя так, как будто вокруг данной корреляционной сферы никакого окружения (т.е. термостата) не существует. Это и следовало ожидать, так как в этом пределе радиус корреляции $R$ оказывается много меньше среднего расстояния между частицами. В результате частицы термостата, находящиеся далеко за пределами корреляционной сферы, уже не могут влиять на то, что происходит внутри этой сферы. Это означает, что столкновения частиц становятся парными.

\section{3. ЭКСПОНЕНЦИАЛЬНАЯ АСИМПТОТИКА}

3.1. Собственные функции уравнения ОЦ. Непосредственной подстановкой в однородное уравнение (9) легко убедиться, что его решением является выражение $\widetilde{w}(r)=\sum_{j} A_{j} e^{-z_{j} r}$, в котором $z_{j}=\lambda_{j}+i \mu_{j}$ являются корнями трансцендентного уравнения

$$
z_{j}=4 \pi \rho \int_{0}^{\infty} C(r) \operatorname{sh}\left(z_{j} r\right) r d r .
$$

Это уравнение имеет один действительный корень $z=\lambda$, значение которого определяется соотношением

$$
\lambda=4 \pi \rho \int_{0}^{\infty} C(r) \operatorname{sh}(\lambda r) r d r
$$


и бесконечное множество комплексных корней $z_{j}=\lambda_{j}+i \mu_{j}$, значения которых могут быть найдены путем решения системы двух уравнений

$$
\begin{aligned}
& \lambda_{j}=4 \pi \rho \int_{0}^{\infty} C(r) \operatorname{sh}\left(\lambda_{j} r\right) \cos \left(\mu_{j} r\right) r d r \\
& \mu_{j}=4 \pi \rho \int_{0}^{\infty} C(r) \operatorname{ch}\left(\lambda_{j} r\right) \sin \left(\mu_{j} r\right) r d r
\end{aligned}
$$

(эта система получается в результате подстановки в (14) $z_{j}=\lambda_{j}+i \mu_{j}$ и приравнивания нулю действительной и мнимой частей). Таким образом, асимптотическое уравнение ОЦ (9) имеет одну действительную собственную функцию $e^{-\lambda r} / r$ и бесконечное множество комплексных собственных функций

$$
\frac{e^{-\lambda_{j} r}}{r} \sin \left(\mu_{j} r\right), \quad \frac{e^{-\lambda_{j} r}}{r} \cos \left(\mu_{j} r\right) .
$$

Соответственно общее решение асимптотического уравнения записывается в виде бесконечного ряда

$$
h=A \frac{e^{-\lambda r}}{r}+\sum_{j=1}^{\infty} \frac{e^{-\lambda_{j} r}}{r}\left[A_{j}^{(1)} \sin \left(\mu_{j} r\right)+A_{j}^{(2)} \cos \left(\mu_{j} r\right)\right] .
$$

Расположим теперь спектр корней $\lambda, \lambda_{j}$ уравнений $(15),(16)$ в порядке их убывания. В зависимости от того, будет ли минимальный корень $\lambda_{\min }=\min \left(\lambda, \lambda_{j}\right)$ равен действительному корню $\lambda$ или одному из комплексных корней $\lambda_{j}$, асимптотика общей корреляционной функции будет определяться одной из формул

$$
h \rightarrow A \frac{e^{-\lambda r}}{r} \quad \text { или } \quad h \rightarrow A_{j} \frac{e^{-\lambda_{j} r}}{r} \cos \left(\mu_{j} r+\psi_{j}\right) .
$$

Первая из них была получена еще Орнштейном и Цернике, вторая была найдена Верле. Их справедливость подтверждается многочисленными данными численного эксперимента, согласно которым экспоненциальная асимптотика может быть как гладкой, так и осциллирующей. При этом граница между той и другой проходит по так называемой линии Уидома-Фишера, положение которой на фазовой диаграмме $\rho \div \theta$ может быть легко воспроизведено по данным численного эксперимента [10]. Но нас в данном случае будет интересовать не столько линия Уидома-Фишера, сколько граница раздела между степенной и экспоненциальной асимптотиками.

3.2. Сходимость интегралов. Согласно (2) в (15), (16) прямую корреляционную функцию $C(r)$ всегда можно записать в виде

$$
C(r)=f(r) e^{w(r)}+\left[e^{w(r)}-w(r)+B[h(r)]\right],
$$

поскольку $h(r)=f(r) e^{w(r)}+e^{w(r)}$, где $f(r)=e^{-\varphi(r)}-1-$ функция Майера. Так как в правой части (19) первое слагаемое $f(r) e^{w(r)} \rightarrow-\varphi(r) \sim 1 / r^{6}$ при $r \rightarrow \infty$, то интегралы в (15), (16) расходятся на бесконечности. Поэтому классическое решение ОЦ $h=A e^{-\lambda r} / r$, надежно подтвержденное данными численного эксперимента, строго говоря, несовместимо с определением прямой корреляционной функции $C(r)=h(r)-w(r)+B[h]$, следующим из распределения Гиббса. 
В литературе было предложено несколько способов устранения этого противоречия [1], [4], [5]. Однако ни один из них не решает и, видимо, не может решить эту проблему. Дело в том, что функция $f(r)$ нулевого порядка по плотности, а бридж-функционал $B[h] \sim \rho^{2}$, поэтому устранить функцию Майера, вызывающую расходимость интегралов, путем соответствующего подбора бридж-функционала в общем виде невозможно.

Видимо, "неустранимость" расходимости интегралов обусловлена тем, что формулы статистической механики определяют параметры системы лишь с экспоненциальной степенью точности. А это предполагает, что мы должны считать $e^{-\lambda r}=0$ не при $r=\infty$, а при некотором конечном значении $r=l$, где величина $l$ должна выбираться из физических соображений. Поэтому при вычислении $\lambda$ вполне закономерно заменить верхний (бесконечный) предел интегрирования в (15), (16) на некоторую конечную величину $r=l$. При таком способе устранения расходимости значения $\lambda$ оказываются сильно зависящими от радиуса обрезания $l$.

Можно, однако, предложить значительно более простой способ устранения расходимости. Он основан на том, что потенциал взаимодействия убывает с расстоянием по закону $\varphi \sim 1 / r^{6}$. При столь быстром убывании функция Майера уже при $r=l \approx 2 \sigma$ оказывается почти на два порядка меньше своего максимального значения в точке $r_{\max }$, в которой $\varphi_{\max } \approx-1$ (эта точка соответствует дну потенциальной ямы). Поэтому начиная с некоторого значения $r=l$ в формуле (19) первым слагаемым можно пренебречь, положив

$$
C(r)= \begin{cases}f(r) e^{w(r)}+\left[e^{w(r)}-w(r)+B[r]\right], & r<l \\ e^{w(r)}-w(r)+B[r], & r>l\end{cases}
$$

Конкретное значение радиуса обрезания $l$ можно найти, например, с помощью следующего условия:

$$
\left|f(l) e^{w(l)}\right|-\left|e^{w(l)}-w(l)+B[l]\right|=0 .
$$

При решении этого уравнения можно воспользоваться уравнением замыкания Мартынова-Саркисова (MC), согласно которому $B=-w^{2} / 2$. В этом случае $e^{w(l)}-w(l)+$ $B[l] \rightarrow w^{3}(l) / 6, f(l) \rightarrow-\varphi(l)$, и уравнение $(21)$ принимает вид $w^{3}(l)=6 \varphi(l)$. Эта оценка, конечно, является достаточно грубой, но она может оказаться полезной.

Учитывая вышесказанное, в дальнейшем будем полагать, что прямая корреляционная функция всегда задается формулой (20).

3.3. Амплитуды асимптотики. В полученных выше формулах значения коэффициента затухания $\lambda$ определяются трансцендентным уравнением (15). Но кроме $\lambda$ формулы (13), (18) содержат также амплитуды $A$ и изотермическую сжимаемость $\kappa_{\theta}$, значения которых еще нужно определить. Начнем с определения амплитуд. 
Асимптотическое уравнение (9) является линейным и однородным. Поэтому оно удовлетворяется при любом произвольном значении $A$. Устранить эту неопределенность можно только с помощью точного уравнения ОЦ (1). Полагая

$$
\widehat{\psi}(k)=4 \pi \int_{0}^{\infty} \psi(r) \frac{\sin (k r)}{k r} r^{2} d r, \quad \psi(r)=\frac{1}{2 \pi^{2}} \int_{0}^{\infty} \widehat{\psi}(k) \frac{\sin (k r)}{k r} k^{2} d k,
$$

где $\psi=h$ или $\psi=C$, запишем его в виде

$$
1+\rho \hat{h}(k)=\frac{1}{1-\rho \widehat{C}(k)}, \quad \rho \hat{h}(k)=\frac{\rho \widehat{C}(k)}{1-\rho \widehat{C}(k)} .
$$

Подставляя найденное таким образом выражение для $\hat{h}(k)$ в обратное преобразование Фурье и вычисляя интеграл

$$
h(r)=4 \pi \rho \int_{0}^{\infty} \frac{\widehat{C}(k)}{1-\rho \widehat{C}(k)} \frac{\sin (k r)}{k r} k^{2} d k
$$

методом теории вычетов [9], получим для общей корреляционной функции $h(r)$ то же выражение (17), которое было получено ранее с помощью асимптотического уравнения ОЦ (9). Более того, значения корней $\lambda, \lambda_{j}, \mu_{j}$ в этой формуле также будут определяться теми же трансцендентными уравнениями (15), (16), которые были найдены ранее. Новыми оказываются только формулы для определения комплексных амплитуд $A_{j}=A_{j}^{(1)}+i A_{j}^{(2)}$

$$
2 \pi \rho A_{j}=\frac{z_{j}^{2}}{4 \pi \rho}\left\{\int_{0}^{\infty} C(r)\left[\operatorname{ch}\left(z_{j} r\right)-\operatorname{sh}\left(z_{j} r\right) /\left(z_{j} r\right)\right] r^{2} d r\right\}^{-1},
$$

где $z_{j}=\lambda_{j}+i \mu_{j}$. В частном случае единственного действительного корня соотношение (25) принимает вид

$$
2 \pi \rho A=\frac{\lambda^{2}}{4 \pi \rho}\left\{\int_{0}^{\infty} C(r)[\operatorname{ch}(\lambda r)-\operatorname{sh}(\lambda r) /(\lambda r)] r^{2} d r\right\}^{-1} .
$$

В случае комплексных корней исходное выражение (25) нужно преобразовать в систему двух уравнений для определения $A_{j}^{(1)}$ и $A_{j}^{(2)}$ путем приравнивания нулю его действительной и мнимой части аналогично выводу трансцендентных уравнений (16).

3.4. Окрестность критической точки. Как известно, изотермическая сжимаемость определяется соотношением

$$
\kappa_{\theta}=\frac{\theta}{\partial P / \partial \rho}=1+4 \pi \rho \int_{0}^{\infty} h(r) r^{2} d r
$$

где $P$ - давление [9]. Подставляя в (27) общую корреляционную функцию $h(r)$ из (17) и интегрируя получившееся выражение по $r$, найдем

$$
\kappa_{\theta}=1+4 \pi \rho \frac{A}{\lambda^{2}}+4 \pi \rho \sum_{j}\left[A_{j}^{(1)} \frac{2 \lambda_{j} \mu_{j}}{\left(\lambda_{j}^{2}+\mu_{j}^{2}\right)^{2}}+A_{j}^{(2)} \frac{\lambda_{j}^{2}-\mu_{j}^{2}}{\left(\lambda_{j}^{2}+\mu_{j}^{2}\right)^{2}}\right] .
$$


При приближении к критической точке эта величина должна стремиться к бесконечности. Это может произойти, только если в формуле (28)

$$
4 \pi \rho \frac{A(\lambda)}{\lambda^{2}} \rightarrow \infty
$$

и радиус корреляции

$$
R=\frac{1}{\lambda} \rightarrow \infty, \quad \lambda \rightarrow 0
$$

остальные члены суммы в (28) остаются конечными при любых значениях $\lambda_{j}, \mu_{j}$. Таким образом, в окрестности критической точки в (17) слагаемое $A e^{-\lambda r} / r$, приводящее к (29), определяет вклад в сжимаемость больших расстояний $r \approx R$, тогда как остальные члены этой формулы описывают состояние корреляционной сферы на малых (по сравнению с $R \rightarrow \infty$ ) расстояниях.

В настоящее время формула $h=A e^{-\lambda r} / r$, полученная еще Орнштейном и Цернике, считается ошибочной, так как она приводит к соотношению (29), из которого, казалось бы, следуют классические критические индексы. Но это предполагает, что константа $A(\lambda) \rightarrow A(0)>0$ при $\lambda \rightarrow 0$. Однако подобное предположение не следует из точного определения амплитуды (26). В следующей работе автор предполагает показать, что на самом деле $A(\lambda) \rightarrow \lambda^{\eta} A_{0} \rightarrow 0$. Поэтому правильное выражение для критической сжимаемости имеет вид

$$
\kappa_{\theta}=4 \pi \rho \frac{A_{0}}{\lambda^{2-\eta}} \rightarrow \infty
$$

что ничему не противоречит. Более того, в той же работе мы покажем, что из формулы $A(\lambda) \rightarrow \lambda^{\eta} A_{0}$ следуют все известные соотношения между критическими индексами. Таким образом, экспоненциальная асимптотика не только определяет линию Уидома-Фишера, но и позволяет описать поведение системы в окрестности критической точки. Добиться таких же результатов с помощью формул, полученных при анализе степенной асимптотики, невозможно. Все это указывает на то, что несмотря на формальную расходимость интегралов экспоненциальная асимптотика на самом деле существует.

\section{4. ГРАНИЦА МЕЖДУ АСИМПТОТИКАМИ}

Объединяя (13) и (18), получим в случае действительного корня

$$
w(r) \rightarrow A \frac{e^{-\lambda r}}{r}-\left(\kappa_{\theta}^{2}-1\right) \varphi(r), \quad h(r) \rightarrow A \frac{e^{-\lambda r}}{r}-\kappa_{\theta}^{2} \varphi(r)
$$

(аналогичное выражение может быть получено и для осциллирующей асимптотики). Отсюда следует, что граница $L$ между экспоненциальной и степенной асимптотиками должна определяться условием обращения в нуль разности

$$
h(L)=A \frac{e^{-\lambda L}}{L}-\kappa_{\theta}^{2} 4 \varepsilon\left(\frac{\sigma}{L}\right)^{6}=0 .
$$


Как известно, любая экспоненциальная функция убывает с расстоянием быстрее любой степенной функции, и, следовательно, на определенном расстоянии степенной "хвост" корреляционных функций всегда должен возникать вместо экспоненциального. Как отмечалось выше, статистическая механика определяет все величины с экспоненциальной степенью точности. Поэтому если подобное явление происходит при $L>l$, где $l$ - расстояние, на котором мы обрезаем потенциал, то, очевидно, что степенной член в (33) мы должны положить равным нулю. Если при этом окажется, что и экспоненциальный член того же порядка малости, что и степенной, то и его мы должны положить равным нулю, т.е. считать, что корреляционные функции на этих расстояниях тождественно равны нулю.

Легко показать, что разложение термического потенциала в ряд по степеням $\rho$ начинается с первой степени, $w \sim \rho$. Поэтому выражение, стоящее в формуле

$$
C(r)=f(r) e^{w(r)}+\left[e^{w(r)}-w(r)+B[r]\right]
$$

в квадратных скобках, пропорционально $w^{3} \sim \rho^{3}$, тогда как первое слагаемое нулевого порядка по $\rho$. Поэтому с точностью до членов порядка $\rho^{3}$ в разреженных газах прямая корреляционная функция всегда равна $C(r) \approx f(r) e^{w(r)}$ (это приближение называется уравнением замыкания Перкуса-Йевика). В результате экспоненциальная асимптотика исчезает, и формула $h \approx \kappa_{\theta}^{2} \varphi(r)$ становится универсальной. С формальной точки зрения это означает, что в разреженных газах $L \leqslant l$.

Противоположная ситуация возникает в окрестности критической точки, где радиус корреляции $R=1 / \lambda \rightarrow \infty$. В этом случае выражение, стоящее в определении функции $C(r)$ в квадратных скобках, стремится к $w^{3} \sim\left(e^{-\lambda r} / r\right)^{3} \rightarrow 1 / r^{3}$, т.е. убывает медленнее, чем потенциал $\varphi \rightarrow 1 / r^{6}$. Поэтому в окрестности критической точки степенной асимптотикой всегда можно пренебречь. Формально это означает, что в этом случае $L \rightarrow \infty$. Поэтому теория критических явлений должна строиться на формулах, описывающих экспоненциальную асимптотику.

\section{5. ЗАКЛЮЧЕНИЕ}

Сформулируем ответы на вопросы, поставленные в разделе 1.

Экспоненциальная асимптотика всегда преобладает на некотором интервале $l<$ $r<L$, где $l \approx 2 \sigma, \sigma$ - диаметр частиц. Но поскольку любая степенная функция на бесконечности убывает медленнее экспоненциальной, на больших расстояниях $r>L$ всегда преобладает степенная асимптотика. Однако, как правило, это происходит на столь больших расстояниях, на которых практически все корреляции исчезают. Поэтому можно положить $L=\infty$ и считать, что в жидкостях на всех расстояниях $r>L$ преобладает экспоненциальная асимптотика. Однако из этого правила существуют два исключения. В газах с понижением плотности $L \leqslant l$. В результате этого область, внутри которой доминирует экспоненциальная асимптотика, исчезает, и на всех расстояниях преобладающей становится степенная асимптотика. Обратная ситуация наблюдается в окрестности критической точки, с приближением к которой $L \rightarrow \infty$. В этом случае степенная асимптотика вообее не играет никакой роли и все определяется экспоненциальной асимптотикой. 


\section{Список литературы}

[1] J. E. Enderby, T. Gaskell, N. H. March, Proc. Phys. Soc., 85 (1965), 217-221.

[2] E. M. Apfelbaum, V.S. Vorob'ev, G. A. Martynov, J. Chem. Phys., 127:6 (2007), 064507.

[3] C. Domb, The Critical Point: A Historical Introduction to the Modern Theory of Critical Phenomena, Taylor and Francis, London, Bristol, 1996.

[4] R. Evans, T. J. Sluckin, J. Phys. C, 14:19 (1981), 2569-2579.

[5] R. J. Leote de Carvalho, R. J. F. Evans, D. C. Hoyle, J. R. Henderson, J. Phys.: Condens. Matter, 6 (1994), 9275-9294.

[6] T. Morita, K. Hiroike, Progr. Theoret. Phys., 23:6 (1960), 1003-1027; 24:2 (1960), 317-330; 25:4 (1961), 537-578.

[7] Г. А. Мартынов, ТМФ, 22:1 (1975), 85-96.

[8] G. A. Martynov, Fundamental Theory of Liquids, Adam Hilger, Bristol, New York, 1992.

[9] Г. А. Мартынов, УФН, 169:6 (1999), 595-624.

[10] Г. Н. Саркисов, УФН, 169:6 (1999), 625-642.

Поступила в редакцию 2.10.2007, после доработки 5.02.2008 